\title{
NASAL CHAMBER CHANGES FOLLOWING RAPID MAXILLARY EXPANSION IN CHILDREN WITH BILATERAL CLEFT LIP AND PALATE
}

\author{
Noha Ibrahim Abdelrahman * and Raghdaa Aboel-Khair Mostafa**
}

\begin{abstract}
Purpose: The aim was to study the skeletal effect of rapid palatal expansion (RPE) on the anterior and posterior nasal width in a sample of Egyptian children (age range 9.4-14.3 years, mean 11.16 years) with bilateral cleft lip and palate (BCLP).

Methods: Twenty Egyptian children with complete BCLP exhibiting maxillary transverse deficiency were divided randomly into; Group 1 received a fixed fan expander and Group 2 a hyrax expander. Cone-beam computed tomography (CBCT) images were taken before $\mathrm{T} 1$ and 3 months after expansion $\mathrm{T} 2$, just before the alveolar cleft grafting (ACG) surgical procedure. Linear and angular measurements were assessed to measure the degree of change on the anterior and posterior nasal chamber widths, dentition, and dental arch.
\end{abstract}

Results: Repeated measure ANOVA with a between variable analysis was used to examine the effect of the two expanders on the dental arch and anterior nasal width as well as posterior nasal width. Statistically significant increase in dentoalveolar measurements were found in each group, nasal widths showed no statistically significant differences before and after expansion.

Conclusion: increase in nasal width were found to be statistically insignificant following RME.

\section{INTRODUCTION}

Individuals born with cleft lip and palate are impacted by this developmental anomaly on several aspects. Clefts of the lip \&/or palate affect the development of the nasomaxillary complex and its neighboring structures in all three planes of space. It commonly causes nasal septum deviation, hypertrophy of the turbinates, and other nasal deformities that result in a reduced nasal airflow capacity, respiratory difficulties and ultimately

\footnotetext{
* Department of Orthodontics, Senior Orthodontist at Cleft Care Clinic affiliated to Oral and Maxillofacial Surgery Department, Faculty of Dentistry, Ain Shams University.

** Department of Oral and Maxillofacial Radiology, Faculty of Dentistry, Ain Shams University.
} 
attributing to oral breathing. Maxillary growth is further complicated by the surgical corrections of the lip and palate performed during the earlier stages after birth. The resultant scar tissue from these surgeries compromises the anterior and posterior nasomaxillary dimensions, usually resulting in a narrow maxillary arch. Rapid palatal expansion has been recognized as an effective treatment method for increasing the maxillary transverse dimensions. Its effect on the upper airway and naso-maxillary complex has also been discussed in the literature for cleft and non cleft individuals. ' With the introduction of the cone beam computed tomography, three dimensional visualization of the nasal chambers and the maxillary arch have been facilitated at a reduced radiation dose and cost when compared to multislice computed tomography. The extent of nasal change associated with RME in Egyptian children with bilateral CLP has not yet been investigated, hence our aim was to understand these possible changes that might occur with two of the most commonly used RME devices in our clinic.

\section{MATERIALS AND METHODS}

This study was approved by the Research Ethical Committee, Faculty of Dentistry, Ain Shams University. A total of 40 pre-operative and postoperative CBCT of patients with complete, nonsyndromic bilateral cleft lip and palate, ages ranging from 9 to 14 years of age, and had received rapid palatal expansion in preparation for the alveolar cleft graft (ACG) procedure. All obtained CBCTs were pre ACG. The sample was grouped into two; Group 1 included children that had received a Fantype fixed maxillary expander, and Group 2 those who had a regular 2-point fixed Hyrax expander. Both expanders were banded to the maxillary first permanent molar with arms extending anteriorly to the most anterior tooth in crossbite; which usually was the deciduous canine, the first deciduous molar, or the first premolar. A semi- rapid expansion protocol with $0.5 \mathrm{~mm}$ expansion per day (daily twice turn of the screw) was adopted with all the participants. All CBCT examinations were acquired using Planmeca ProMax ${ }^{\circledR}$ 3D Classic (Helsinki, Finland), with Exposure parameters: $120 \mathrm{kVp}$, $0.4 \mathrm{~mm}$ voxel size and $13.7 \mathrm{sec}$ Scanning time with Field of View (FOV) $23 \times 17 \mathrm{~cm}$. The scans were taken with the patients in upright position, Frankfort horizontal plane parallel to the floor which was adjusted via the laser beams of the machine. The scans were obtained with the tongue resting on the palate. Swallowing and breathing were evaded during the scan. CBCT scans were acquired immediately before receiving the expanders (T1) and 3 months after concluding expansion and just before the ACG surgery (T2). Linear and angular measurements were done pre and post maxillary expansion via an oral and maxillofacial radiologist who had no previous contact with any of the participating subjects to prevent bias.

\section{Measurements were as follows;}

Anterior nasal arch width (ANW) was measured on the coronal slice as the widest distance of the nasal aperture. This coronal slice was standardized at the level of the nasolacrimal canal on the axial slice. Using the same anterior coronal slice previously obtained to measure the ANW, the anterior interarch distance was measured at the level of the CEJ of the present tooth buccally to the contralateral side. Fig.1.

Posterior nasal width (PNW) was measured on coronal slice as the widest distance of the nasal aperture. This was measured on a coronal slice after viewing them sequentially, the slice that clearly demonstrated the apices of the mesiobuccal and distobuccal roots of both the right and left first molars was chosen. Fig.2.

Using the same coronal slice previously obtained, the Intermolar Alveolar Crest width was measured at the level of the CEJ of the first molar buccally to the contralateral side. 


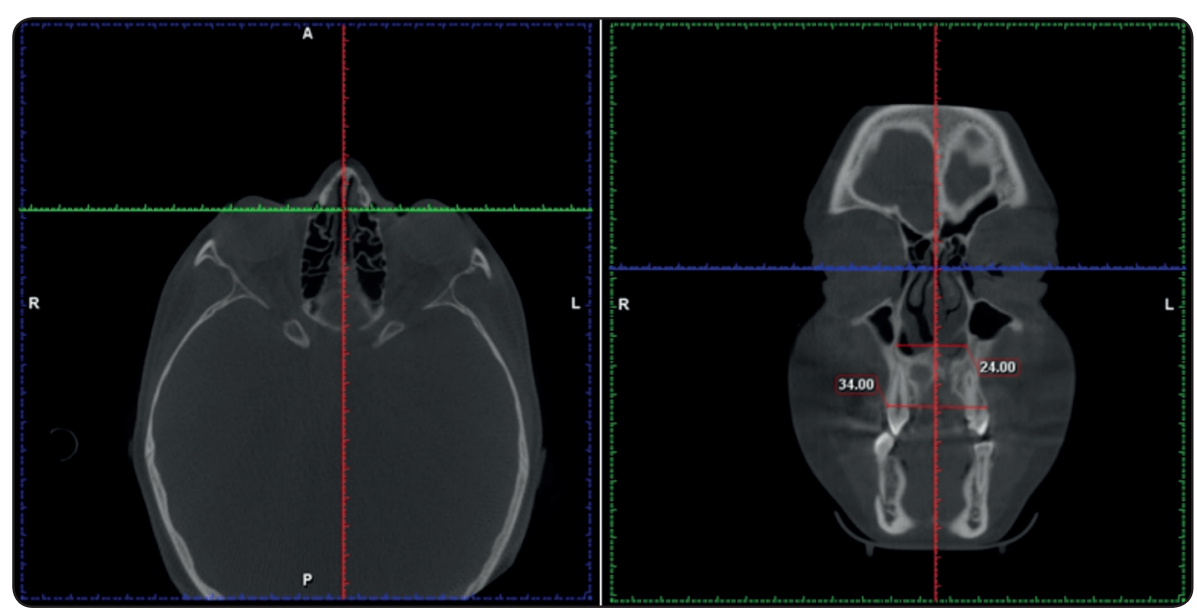

Fig. (1) Showing the axial slice at the level of the nasolacrimal canal, the corresponding coronal slice to measure ANW and anterior Inter-arch distance.

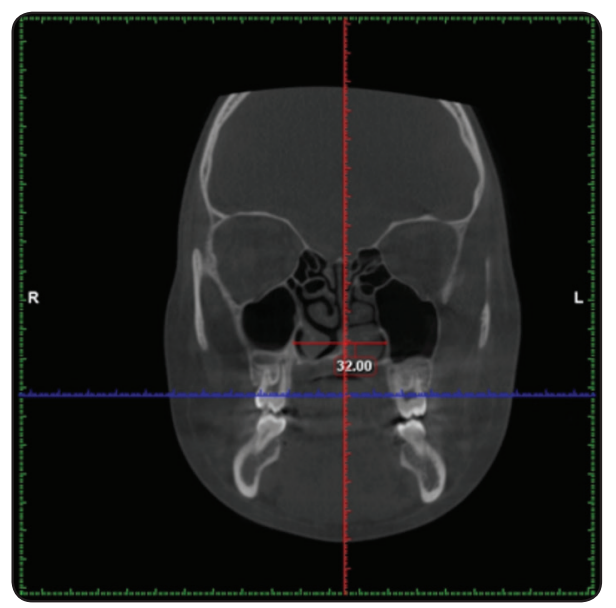

Fig. (2) Posterior nasal width (PNW)
Degree of dental tipping was evaluated by measuring two angles;

Buccal molar tipping: was measured by an angle created between two lines extending from the most inferior point of the crista galli vertex and extending to the CEJ point on the buccal surfaces of the maxillary first molars, bilaterally. While the palatal molar tipping was measured by an angle from the same point on the crista galli vertex and extending to the CEJ point on the palatal surfaces of the maxillary first molars, bilaterally. Fig.3

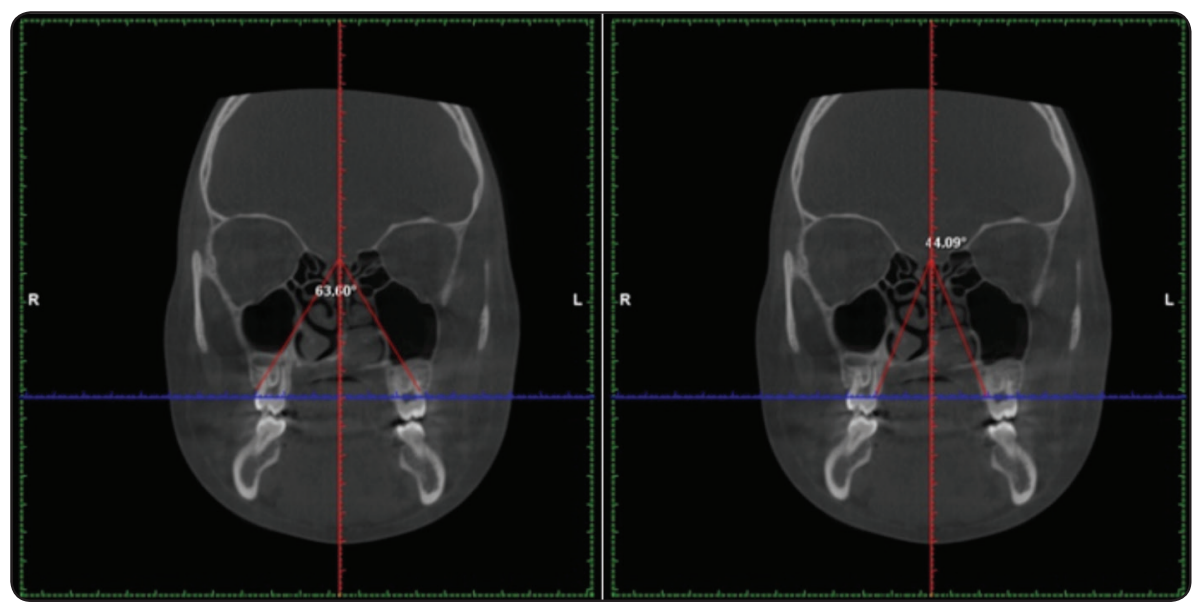

Fig. (3) Showing buccal and platal molar tipping angles. 


\section{Posterior inter-arch distance}

The axial slice corresponding to the coronal slice previously mentioned and passing at the level of the CEJ bilaterally was used. Posterior inter-arch distance was measured as the maximum distance between the buccal surfaces of the crowns of the maxillary first molars. Fig. 4

All measurements were blindly measured at T1 and $\mathrm{T} 2$, by an oral and maxillofacial radiologist. Then all parameters were re-measured two weeks later. The mean of the two readings was calculated and used for further analysis

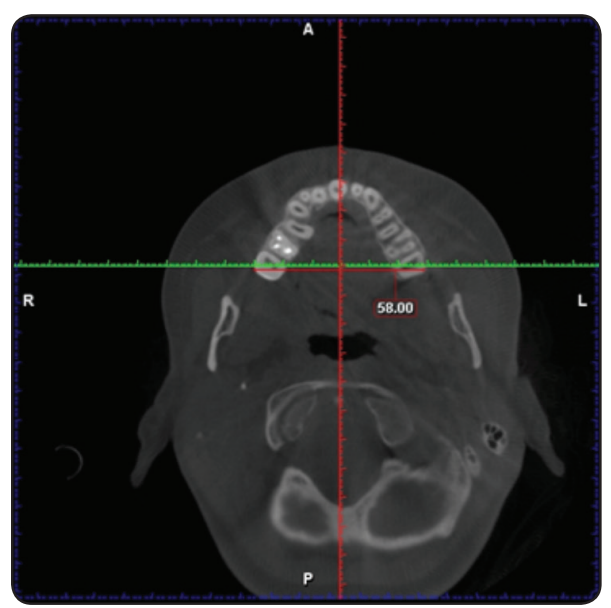

Fig. (4) Posterior inter arch distance.

\section{RESULTS}

Repeated measure ANOVA with a between variable analysis was used to examine the effect of the two expanders and time at $\mathrm{T} 1$ and $\mathrm{T} 2$ on different linear and angular parameters.

TABLE (1) Mean and standard deviation of the linear and angular parameters measured at the baseline and 3 month follow up in both groups.

\begin{tabular}{|c|c|c|c|c|}
\hline \multirow{2}{*}{ Measurement } & \multicolumn{2}{|c|}{ Group 1 (Fax Expander) } & \multicolumn{2}{c|}{ Group 2 (Hyrax Expander) } \\
\cline { 2 - 5 } & $\mathrm{T} 1$ & $\mathrm{~T} 2$ & $\mathrm{~T} 1$ & $\mathrm{~T} 2$ \\
\hline Anterior Nasal Width (ANW) & $26.38 \pm 3.08$ & $27.34 \pm 2.72$ & $25.26 \pm 4.13$ & $25.37 \pm 7.21$ \\
\hline Anterior Inter-arch distance & $31.65 \pm 2.93$ & $41.62 \pm 3.6$ & $30.03 \pm 2.3$ & $41.66 \pm 2.65$ \\
\hline Posterior Nasal Width (PNW) & $28.92 \pm 3.18$ & $28.94 \pm 3.51$ & $29.86 \pm 4.61$ & $31.03 \pm 4.15$ \\
\hline Palatal molar tipping angle & $33.08 \pm 4.7$ & $35.68 \pm 3.75$ & $33.68 \pm 5.25$ & $38.52 \pm 6.36$ \\
\hline Buccal molar tipping angle & $51.71 \pm 5.87$ & $56.71 \pm 5.3$ & $53.21 \pm 7.58$ & $57.69 \pm 7.84$ \\
\hline Inter-molar Alveolar Crest width & $51.71 \pm 5.87$ & $55.92 \pm 3.63$ & $53.21 \pm 7.58$ & $58 \pm 3.86$ \\
\hline Posterior inter-arch distance & $52.7 \pm 3.05$ & $53.84 \pm 2.85$ & $51.23 \pm 3.83$ & $54.64 \pm 3.6$ \\
\hline
\end{tabular}

Regarding time of measurements effect, there was a statistically significant difference in anterior interarch distance, palatal molar tipping angle, buccal molar tipping angle, Intermolar Alveolar Crest width and Posterior inter-arch distance between time at baseline and the 3 month follow up, all of them showed significant increase between the two points of time. Table 1,2.

Regarding the type of treatment in the 2 groups, all measured parameters showed non statistical differences in each time between the two groups (table 2). 
TABLE (2) The main effect of the groups, time of measurements and the interaction effect between time of measurement and groups

\begin{tabular}{|c|c|c|c|c|c|c|}
\hline \multirow{2}{*}{ Parameters } & \multicolumn{2}{|c|}{$\begin{array}{c}\text { Group } \\
\text { (type of expander) }\end{array}$} & \multicolumn{2}{|c|}{ Time } & \multicolumn{2}{|c|}{ Group*Time } \\
\hline & $\mathrm{F}$ & Sig. & $\mathrm{F}$ & Sig. & $\mathrm{F}$ & Sig. \\
\hline Anterior Nasal Width (ANW) & 0.488 & 0.498 & 0.235 & 0.637 & 0.145 & 0.710 \\
\hline Anterior Inter-arch distance & 0.407 & 0.535 & 130.750 & 0.000 & 0.774 & 0.396 \\
\hline Posterior Nasal Width (PNW) & 0.561 & 0.468 & 1.335 & 0.270 & 1.220 & 0.291 \\
\hline palatal molar tipping angle & 0.475 & 0.504 & 11.378 & 0.006 & 1.043 & 0.327 \\
\hline Buccal molar tipping angle & 0.147 & 0.708 & 8.966 & 0.011 & 0.028 & 0.871 \\
\hline Intermolar Alveolar Crest width & 0.558 & 0.469 & 7.114 & 0.021 & 0.028 & 0.869 \\
\hline Posterior inter-arch distance & 0.041 & 0.844 & 11.783 & 0.005 & 2.950 & 0.112 \\
\hline
\end{tabular}

\section{DISCUSSION}

Efficacy of the RME on expanding the dental arches in non-cleft as well as cleft individuals has been well established ${ }^{5-8}$ and is considered routine treatment during multidisciplinary management of individuals with complete CLP. Most children with BCLP require RME at some point, due to the high incidence of anterior collapse of the arch following primary lip and primary palate surgical corrections $\mathrm{s}^{3,4}$, which is usually addressed in our clinic by using the fan expander when more anterior expansion is required that posterior, and the hyrax expander when significant anterior as well as posterior expansion are required. Usually this occurs in preparation for the alveolar cleft grafting procedure during the mixed dentition/early permanent dentition stages. The effect of the RME on the nasal chambers were raised with a considerable amount of debate whether the change produced by RME increases the nasal chambers width ${ }^{9-11}$, which inevitably will enhance nasal breathing in these individuals, otherwise suffering nasal respiration restriction. Our study's purpose was to assess whether the type of expander would affect the anterior and posterior nasal widths after expanding the maxillary dental arch. The anterior and posterior nasal widths were unaffected by the type of the expander, a difference was found between the pre-expansion widths and the post-expansion ones, however, this difference was clinically rather that statistically significant. Several guardians of the participating children reported a reduced hyper-nasality tone during speech and more facilitated nasal breathing. Further studies measuring with acoustic rhinometry, as well as overall three-dimensional volumetric changes of the nasal passages and airway might be beneficial to statistically analyze the skeletal effects of the different expanders on the nasal chambers.

\section{CONCLUSION}

Both the Hyrax and the fixed Fan expanders showed significant dentoalveolar effects on the maxillary arch, however nasal width changes following expansion did not show statistically significant changes. 


\section{REFERENCES}

1. Bardach J., Cutting C. Anatomy of the unliateral and bilateral cleft lip and nose. In: Bardach J, Morris Hl, ed. Multidisciplinary Management of Cleft Lip and Palate. Philadelphia: WB Saunders; 1990.

2. Warren DW, Drake AF. Cleft nose: form and function. Clin Plast Surg. 1993;20:769-779.

3. Wetmore RF. Importance of maintaining normal nasal function in the cleft palate patient. Cleft Palate Craniofac J. 1992;29:498-506.

4. Silva Filho OG, Ramos AL, Abdo RCC. The influence of unilateral cleft lip and palate on maxillary dental arch morphology. Angle Orthod. 1992;62:183-190.

5. Capelozza I, DeAlmeida A, Ursi W. Rapid maxillary expansion in cleft lip and palate patients. J Clin Orthod 1994; 28: 34-9.

6. Weissheimer A, Menezes L, Mezomo M, Dias D, Lima E, Rizzatto S. Immediate effects of rapid maxillary expansion with Haas-type and hyrax-type expanders: a randomized clinical trial. Am J Orthod Dentofacial Orthop. 2011; 140(3) :366-76.

7. Figueiredo D, Bartolomeo F, Romualdo C, Palomo J, Horta C, Andrade I, et al. Dentoskeletal effects of 3 maxillary expanders in patients with clefts: a cone-beam computed tomography study. Am J Orthod Dentofacial Orthop. 2014; 146(1): 73-81.

8. Trindade-Suedam IK, Castilho RL, Sampaio-Teixeira ACM, Araujo BMAM, Fukushiro AP, Campos LD, Trindade IEK. Rapid Maxillary Expansion Increases Internal Nasal Dimensions of Children with Bilateral Cleft Lip and Palate. Cleft Palate Craniofac J. 2016; 53(3):272-277.

9. Mordente CM, Palomo JM, Horta MCR, Souki BQ, Oliveira DD, Andrade I. Upper airway assessment using four different maxillary expanders in cleft patients: A cone-beam computed tomography study. Angle Orthod. 2016;86:617-624. 